\title{
MODELO PARA PREVISÃO DE POSIÇÃO DE OBJETOS EM 3D
}

\author{
Jair Agner Júnior \\ Bruno Schulze \\ Mariza Ferro \\ Laboratório Nacional de Computação Científica \\ \{jairagnerjunior; bruno.schulze; mariza.ferro\}@gmail.com \\ Ernesto Rademaker Martins \\ Marcos dos Santos \\ Centro de Análises de Sistemas Navais \\ \{radmart; marcosdossantos_doutorado_uff $\} @$ yahoo.com.br
}

\section{RESUMO}

A previsão de posição de objetos em 3D é um processo complexo, o qual exige algoritmos de associação capazes de lidar com o uso de técnicas de filtragem lineares e não lineares. Os modelos utilizados em sistemas de controle de tráfego aéreo, por serem desenvolvidos em duas dimensões, não são adequados para aplicações com alvos militares devido às variações de altitude que esses objetos desenvolvem. Dessa forma, a partir das informações de posições de um objeto no espaço obtidas por meio de um dado sensor, foi utilizado um método para se deduzir o valor da velocidade do mesmo, de modo a compor o vetor de medidas modelado. Com os dados desse vetor é calculada a próxima posição do objeto medido de forma tridimensional. Esse modelo pode ser implementado em sistemas tanto civis como militares, permitindo previsão de posição de objetos 3D em tempo real. Adotou-se como estimadores de estado o Filtro de Kalman Linear e o Filtro de Kalman Estendido com integração através do agregador de filtros conhecido como IMM (Múltiplos Modelos Interagentes). Exemplos numéricos ilustram a aplicabilidade e desempenho do método proposto.

Palavra-chave: Posição de Objetos em 3D; Filtro de Kalman; Múltiplos Modelos Interagentes; Movimento Curvilíneo Planar.

\begin{abstract}
$3 \mathrm{D}$ object position prediction is a complex process that requires association algorithms that can handle the use of linear and nonlinear filtering techniques. The models used in air traffic control systems, for being developed in two dimensions, are not suitable for applications with military targets due to the altitude variations of these objects. Thus, from the position information of an object in space obtained through a given sensor, in this work a method was used to deduce its velocity value, in order to compose the modeled measurement vector. With the data from this vector, the next position of
\end{abstract}


the three-dimensional measured object is calculated. This model can be implemented in both civil and military systems, allowing real-time 3D object position prediction. As state estimators were adopted the Linear Kalman Filter and the Extended Kalman Filter with integration through the filter aggregator known as IMM (Interacting Multiple-Model). Numerical examples illustrate the applicability and performance of the proposed method.

Keywords: 3D Object Position; Kalman Filter; Interacting Multiple-Model; Planar Curvilinear Motion.

\section{Como Citar:}

AGNER, Júnior; SCHULZE, Bruno; FERRO, Mariza; RADEMAKER, Ernesto; SANTOS, Marcos. Modelo para Previsão de Posição de Objetos em 3D. In: SIMPÓSIO DE PESQUISA OPERACIONAL E LOGÍSTICA DA MARINHA, 19., 2019, Rio de Janeiro, RJ. Anais [...]. Rio de Janeiro: Centro de Análises de Sistemas Navais, 2019.

\section{INTRODUÇÃO}

A proposta deste trabalho é o desenvolvimento de um modelo com foco na previsão de posição de objetos em 3D, tendo como base o descrito em [1]. Dessa forma, a partir das informações de posições de um objeto no espaço obtidas por meio de um dado sensor, foi utilizado um método para se deduzir o valor da velocidade do mesmo, de modo a compor o vetor de medidas modelado. Com os dados desse vetor, buscou-se calcular a próxima posição do objeto medido. O problema de previsão de posição de objetos manobrantes não é uma tarefa trivial, pois exige diversos estudos e análises variadas, não só de ordem conceitual, mas também de ordem prática. Diversos modelos têm sido desenvolvidos para aprimorarem as soluções existentes, como os descritos em [2], [3], 4], entre outros.

A previsão de posição de objetos tem como base a teoria da estimação, que possui o propósito de desenvolver estimadores, também conhecidos como filtros, os quais tenham a possibilidade de serem aplicados em problemas de engenharia, tais como estimação de órbita e altitude, sistemas de potência, detecção de falhas, vigilância e determinação do posicionamento futuro de alvos por radar ou sonar, navegação integrada, entre outros [5]. Neste contexto, procura-se estimar as variáveis de estado de um sistema estocástico linear ou não linear através da filtragem. Dessa forma, são desenvolvidos modelos para serem utilizados nas filtragens, que buscam representar essas variáveis de estado de maneira aproximada, o que permite obter-se uma solução para estimação das posições futuras de objetos em movimento.

Dentre a grande variedade de modelos dinâmicos existentes foram escolhidos dois para a elaboração deste trabalho, os quais tratam de objetos em deslocamento: o modelo com Velocidade Constante (CV), implementado com o Filtro de Kalman (FK) para tratar da filtragem linear e o modelo de Giro Constante (CT), implementado com o Filtro de Kalman Estendido (FKE) para cobrir a filtragem não-linear. Os modelos CV e CT representam casos particulares do modelo de cinemática do movimento curvilíneo planar (CLM) com dinâmica de movimento bidimensional [6].

Para utilizar mais de um modelo em um problema de estimação de estados, deve-se fazer uso de uma técnica que permita a utilização de modelos em paralelo. A abordagem adotada para trabalhar com múltiplos modelos paralelamente foi o algoritmo 
IMM, proposto por [7].

\section{MÚLTIPLOS MODELOS INTERAGENTES (IMM)}

O IMM estima o estado de um sistema dinâmico e sua respectiva matriz de covariância através da soma ponderada das estimativas de $\mathrm{N}$ modelos de FK executados em paralelo. É uma ferramenta de estimação que em cada iteração são calculados os fatores de ponderação para a combinação das estimativas de cada um dos modelos de filtro.

A implementação do IMM foi necessária, pois um objeto se deslocando no espaço pode desenvolver diferentes padrões de comportamento ao longo do tempo, e o uso de somente um filtro se torna ineficiente para se adequar a essas variações de comportamento. Assim, foi utilizado o IMM para trabalhar de forma simultânea com o FK (utilizando o modelo CV) e com o FKE (utilizando o modelo CT).

\section{MODELOS MATEMÁTICOS}

Muitas das técnicas de previsão de posicionamento de objetos são descritas através de modelos, os quais baseiam-se nos aspectos de comportamento e observações do alvo. O comportamento é normalmente representado na forma de um modelo dinâmico ou de movimento, também chamado de modelo de estados, descrevendo a evolução de diversas grandezas físicas, como posição, velocidade, etc. O aspecto que trata das observações do objeto é representado através de modelos de observação [8]. Dessa forma, para tratar adequadamente o problema de previsão de posição, são descritos os modelos matemáticos, levando em conta as equações, variáveis e parâmetros pertinentes.

As Equações (1) e (2) definem o FK e o FKE, que são descritos de forma detalhada com aplicações em [5], [9] e [10]. Entretanto, neste trabalho o filtro de Kalman soluciona o problema geral de buscar estimar o estado $\mathbf{x} \in \mathbf{R}^{8}$ de um processo controlado em tempo discreto que é governado pela equação de diferença estocástica linear (1), com uma medição $\mathbf{z}_{k} \in \mathbf{R}^{5}(2)$ descritas por:

$$
\begin{gathered}
\mathbf{x}_{k+1}=F_{k} \mathbf{x}_{k}+G_{k} \mathbf{u}_{k}+B_{k} \mathbf{w}_{k}, \\
\mathbf{z}_{k+1}=H_{k+1} \mathbf{x}_{k+1}+\mathbf{v}_{k+1}
\end{gathered}
$$

A estimação de estados efetuada pela Equação (1) obedece as leis que governam o movimento do objeto que está em deslocamento. Essas leis, assim como a abordagem adotada para o cálculo das medições efetuadas pela Equação (2), serão descritas mais adiante. As variáveis aleatórias $\mathbf{w}_{k}$ e $\mathbf{v}_{k+1}$ representam o ruído de processo e o ruído de medição, respectivamente. Elas são consideradas ruídos tipo branco (gaussiano), independentes, com distribuição de probabilidade contínua, normal, identicamente distribuídas, com média zero e covariância $Q$ e $R$, respectivamente. Assim, $p(\mathbf{w}) \sim N(0, Q)$ e $p(\mathbf{v}) \sim N(0, R)$. A matriz $F_{8 \times 8}$ de transição de estados, na Equação (1), refere-se ao estado da amostra no intervalo de tempo anterior $k$, para o estado atual no tempo $k+1$, na ausência de uma função de condução ou ruído do processo. A matriz $G_{8 \times 5}$ refere-se a entrada de controle opcional $\mathbf{u}_{k} \in \mathbf{R}^{5}$ para o estado $\mathbf{x}_{k+1}$. Nesta abordagem, a entrada de controle $\mathbf{u}_{k}$ pode ser atribuída como o ruído da posição do sensor que faz a medição da posição do objeto. Dessa forma, o movimento do sensor tem influência sobre a posição do objeto. A matriz $G_{8 \times 5}$ 
controla os efeitos de $\mathbf{u}_{k}$, e como neste trabalho é considerado um observador estático, o termo $G_{k} \mathbf{u}_{k}$ não é incorporado na equação do processo. A matriz $B_{8 \times 5}$ controla os efeitos de $\mathbf{w}_{k}$. A matriz $H_{5 \times 8}$, na Equação de medição (2), relaciona o estado à medida $\mathbf{z}_{k+1}$.

Para desenvolver esse trabalho, era necessário adotar um modelo de observações para o objeto a ser estudado. Assim, foi utilizado como referência as medidas de um sistema radar simulado e o objeto como sendo uma aeronave (alvo). Essas medidas foram representadas na forma do vetor $\mathbf{z}_{k}=\left[\begin{array}{lll}\mathbf{r}_{k} & \theta_{k} & \phi_{k}\end{array}\right]^{T}$, onde $\mathbf{r}_{k}$ é a distância em metros do objeto ao observador, $\theta_{k}$ é o ângulo de azimute em graus medido desde o norte geográfico ao centro do objeto e $\phi_{k}$ é o ângulo de elevação em graus medido desde um referencial horizontal nulo (plano horizontal, no caso nível do mar) ao centro do objeto. Sabe-se que estas medidas são perturbadas por ruídos (ou erros), em distância $\left(\mathbf{v}_{k}^{d}\right)$, no ângulo de azimute $\left(\mathbf{v}_{k}^{\theta}\right)$ e no ângulo de elevação $\left(\mathbf{v}_{k}^{\phi}\right)$, os quais são assumidos independentes e estacionários com distribuição Gaussiana de média zero e variâncias constantes $\sigma_{d}, \sigma_{\theta} \mathrm{e}$ $\sigma_{\phi}$, respectivamente.

O objeto de estudo foi tratado de forma pontual, descrito em três dimensões, com vetor posição representado por $\mathbf{r}(t)=[\mathbf{x}(t) \mathbf{y}(t) \mathbf{h}(t)]^{T}$, em coordenadas cartesianas. Para o modelo dinâmico ou de movimento, foram utilizadas como variáveis de estado $x, y$ (coordenadas horizontais), $h$ (altitude), $\chi$ (ângulo de proa ou rumo - horizontal), $\gamma$ (ângulo de trajetória - vertical), v (vetor velocidade) e a (vetor aceleração). Essas variáveis podem ser visualizadas na Figura 1 .
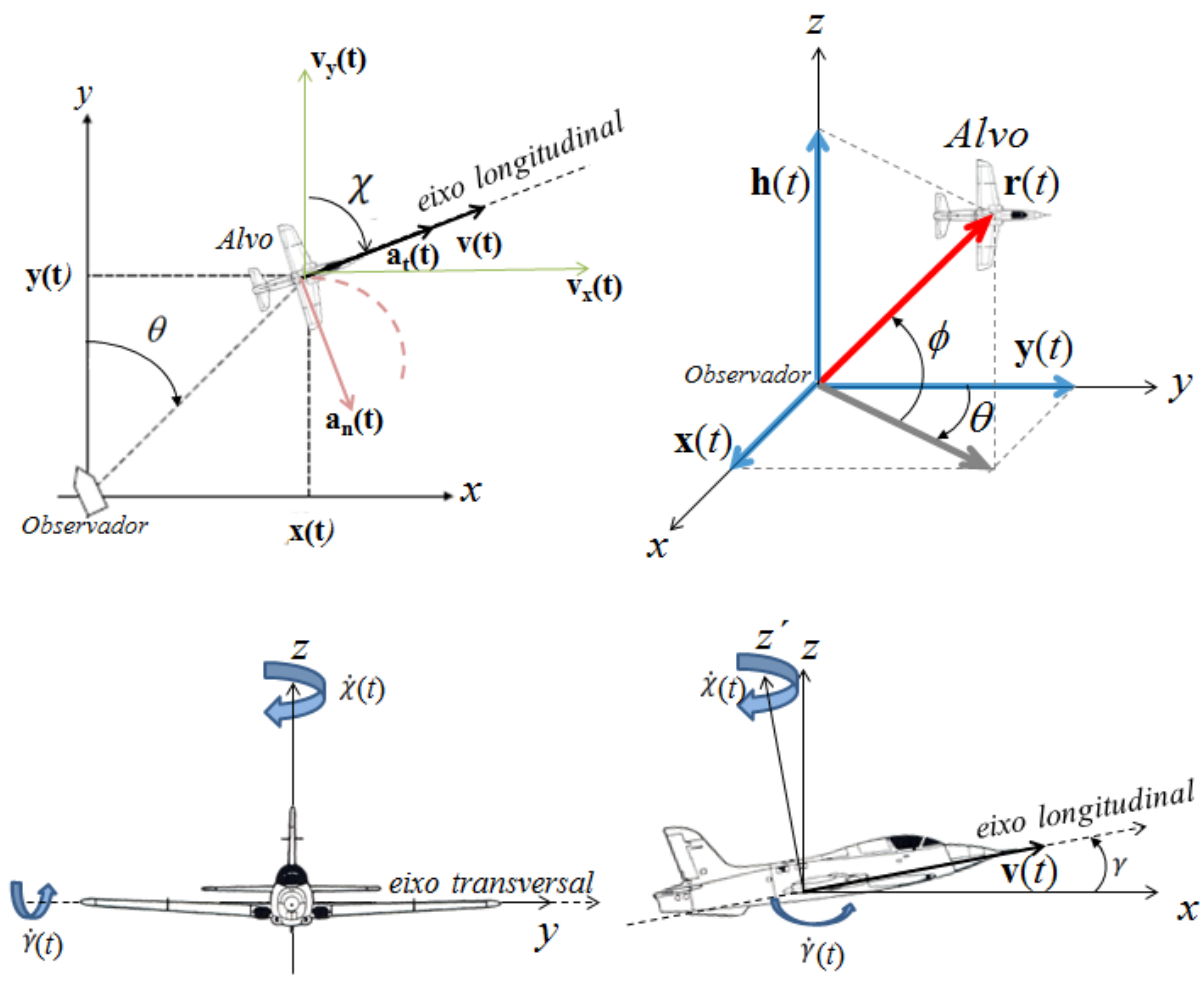

Figura 1: Ilustração das variáveis.

As equações (3), (4) e (5) são obtidas a partir da derivada das componentes de $\mathbf{r}(t)$ e descrevem a velocidade do objeto em função das coordenadas cartesianas relacionando 
a velocidade tangencial instantânea $\mathbf{v}(t), \gamma$ e $\chi$.

$$
\begin{gathered}
\mathbf{v}_{x}(t)=\mathbf{v}(t) \cdot \cos (\chi(t)) \cdot \cos (\gamma(t)) \\
\mathbf{v}_{y}(t)=\mathbf{v}(t) \cdot \sin (\chi(t)) \cdot \cos (\gamma(t)) \\
\mathbf{v}_{h}(t)=\mathbf{v}(t) \cdot \sin (\gamma(t))
\end{gathered}
$$

A diferenciação das equações (3), (4) e (5) resultam em equações diferenciais de segunda ordem, que rearranjadas podem ser reescritas respectivamente por (6), (7) e (8):

$$
\begin{gathered}
\mathbf{a}_{x}(t)=\dot{\mathbf{v}}(t) \cdot \cos (\chi(t)) \cdot \cos (\gamma(t))-\mathbf{v}_{y}(t) \cdot \dot{\chi}(t)-\mathbf{v}_{h}(t) \cdot \cos (\chi(t)) \cdot \dot{\gamma}(t) \\
\mathbf{a}_{y}(t)=\dot{\mathbf{v}}(t) \cdot \sin (\chi(t)) \cdot \cos (\gamma(t))+\mathbf{v}_{x}(t) \cdot \dot{\chi}(t)-\mathbf{v}_{h}(t) \cdot \dot{\gamma}(t) \\
\mathbf{a}_{h}(t)=\dot{\mathbf{v}}(t) \cdot \sin (\gamma(t))+\frac{\mathbf{v}_{y}(t)}{\sin (\chi(t))} \cdot \dot{\gamma}(t)
\end{gathered}
$$

Sabendo que $\dot{\chi}(t)$ é a taxa de variação angular horizontal e $\dot{\gamma}(t)$ é a taxa de variação angular vertical do objeto em deslocamento espacial.

Para o modelo, foi considerado a atuação da aceleração tangencial $\mathbf{a}_{t g}(9)$, que é tangencial ao movimento e provoca a variação do módulo da velocidade do corpo e da aceleração centrípeta $\mathbf{a}_{c p}$, que é perpendicular ao movimento e modifica a direção da velocidade, possuindo uma componente horizontal $\mathbf{a}_{c p \chi}$ e outra vertical $\mathbf{a}_{c p \gamma}$ (11).

$$
\begin{gathered}
\mathbf{a}_{t g}=\dot{\mathbf{v}}(t), \\
\mathbf{a}_{c p \chi}=\dot{\mathbf{v}}(t) \cdot \dot{\chi}(t)=\dot{\mathbf{v}}(t) \cdot \omega_{\chi}(t) \\
\mathbf{a}_{c p \gamma}=\dot{\mathbf{v}}(t) \cdot \dot{\gamma}(t)=\dot{\mathbf{v}}(t) \cdot \omega_{\gamma}(t)
\end{gathered}
$$

\subsection{Dedução dos Modelos CV e CT}

Baseado em [6], que descreve o modelo CLM em duas dimensões, foi realizada a dedução para os dois modelos 3D aqui apresentados. No modelo CV o objeto se movimenta com velocidade constante e em linha reta. Dessa forma, $\mathbf{v}_{x}(t), \mathbf{v}_{y}(t)$ e $\mathbf{v}_{h}(t)$ são constantes, e $\mathbf{a}_{t g}, \mathbf{a}_{c p \chi}$ e $\mathbf{a}_{c p \gamma}$ são nulas. Consequentemente, $\mathbf{a}_{x}(t), \mathbf{a}_{y}(t)$ e $\mathbf{a}_{h}(t)$ são nulas.

No modelo CT o movimento se dá com velocidade e taxa de giro constantes. Assim, $\mathbf{v}_{x}(t), \mathbf{v}_{y}(t)$ e $\mathbf{v}_{h}(t)$ são constantes. A $\mathbf{a}_{t g}, \mathbf{a}_{c p \chi}$ e $\mathbf{a}_{c p \gamma}$, são dadas respectivamente por (12), (13) e (14). A partir das mesmas obtemos $\mathbf{a}_{x}(t), \mathbf{a}_{y}(t)$ e $\mathbf{a}_{h}(t)$, que são dadas respectivamente por (15), (16) e (17).

$$
\begin{gathered}
\mathbf{a}_{t g}=\dot{\mathbf{v}}(t)=0 \\
\mathbf{a}_{c p \chi}=\mathbf{v}(t) \cdot \omega_{\chi}(t)=c t e \\
\mathbf{a}_{c p \gamma}=\mathbf{v}(t) \cdot \omega_{\gamma}(t)=c t e \\
\mathbf{a}_{x}(t)=-\mathbf{v}_{y}(t) \cdot \omega_{\chi}(t)-\mathbf{v}_{h}(t) \cdot \cos (\chi(t)) \cdot \omega_{\gamma}(t) \\
\mathbf{a}_{y}(t)=\mathbf{v}_{x}(t) \cdot \omega_{\chi}(t)-\mathbf{v}_{h}(t) \cdot \omega_{\gamma}(t) \\
\mathbf{a}_{h}(t)=\frac{\mathbf{v}_{y}(t)}{\sin (\chi(t))} \cdot \omega_{\gamma}(t)
\end{gathered}
$$


Passando a trabalhar com a Equação (1) em tempo contínuo e derivando a mesma em função do tempo, temos a equação vetorial de processo em tempo contínuo (18):

$$
\dot{X}(t)=A(t) \cdot X(t)+U
$$

Em (18), $X(t)$ é o vetor de estados definido por 19$)$ e $\dot{X}(t)$ é definido por 20$). A(t)$ é a matriz auxiliar de dedução da matriz de transição de estados $F$ da Equação (1). Face a limitação de espaço, foi suprimido o " $(t)$ " dos componentes dos vetores $X(t)$ e $\dot{X}(t)$ :

$$
\begin{gathered}
X(t)=\left[\begin{array}{llllllll}
\mathbf{x} & \mathbf{v}_{x} & \mathbf{y} & \mathbf{v}_{y} & \mathbf{h} & \mathbf{v}_{h} & \omega_{\chi} & \omega_{\gamma}
\end{array}\right]^{T} \\
\dot{X}(t)=\left[\begin{array}{llllllll}
\dot{\mathbf{x}} & \mathbf{a}_{x} & \dot{\mathbf{y}} & \mathbf{a}_{y} & \dot{\mathbf{h}} & \mathbf{a}_{h} & 0 & 0
\end{array}\right]^{T}
\end{gathered}
$$

O ruído de processo em tempo contínuo $(U)$, pode ser considerado como alterações na velocidade e na taxa de giro, fruto de pequenas perturbações. Dessa forma, foi adotado 21) para o Modelo CV e 22) para o Modelo CT:

$$
\begin{aligned}
& U=\left[\begin{array}{llllllll}
0 & 1 & 0 & 0 & 0 & 0 & 0 & 0 \\
0 & 0 & 0 & 1 & 0 & 0 & 0 & 0 \\
0 & 0 & 0 & 0 & 0 & 1 & 0 & 0 \\
0 & 0 & 0 & 0 & 0 & 0 & 0 & 0 \\
0 & 0 & 0 & 0 & 0 & 0 & 0 & 0
\end{array}\right]^{T} \cdot \mathbf{w}, \\
& U=\left[\begin{array}{llllllll}
0 & 1 & 0 & 0 & 0 & 0 & 0 & 0 \\
0 & 0 & 0 & 1 & 0 & 0 & 0 & 0 \\
0 & 0 & 0 & 0 & 0 & 1 & 0 & 0 \\
0 & 0 & 0 & 0 & 0 & 0 & 1 & 0 \\
0 & 0 & 0 & 0 & 0 & 0 & 0 & 1
\end{array}\right]^{T} \cdot \mathbf{w}
\end{aligned}
$$

w é o vetor do ruído em cada um dos eixos cartesianos $\left(w_{x}, w_{y}\right.$ e $\left.w_{h}\right)$ e nas taxas de giro $\left(w_{\chi}\right.$ e $\left.w_{\gamma}\right)$, sendo dado por: $\mathbf{w}=\left[\begin{array}{lllll}w_{x} & w_{y} & w_{h} & w_{\chi} & w_{\gamma}\end{array}\right]^{T}$.

A matriz $A$, para o modelo CV é obtida substituindo (19), (20) e 21 em (18), sendo dada por (23), e para o modelo CT, substituindo (19), (20) e (22) em (18) sendo dada por (24):

$$
\begin{gathered}
\quad\left[\begin{array}{cccccc}
0 & 1 & 0 & 0 & 0 & 0 \\
0 & 0 & 0 & 0 & 0 & 0 \\
0 & 0 & 0 & 1 & 0 & 0 \\
0 & 0 & 0 & 0 & 0 & 0 \\
0 & 0 & 0 & 0 & 0 & 1 \\
0 & 0 & 0 & 0 & 0 & 0
\end{array}\right] \\
{\left[\begin{array}{cccccccc}
0 & 1 & 0 & 0 & 0 & 0 & 0 & 0 \\
0 & 0 & 0 & -\omega_{\chi}(t) & 0 & -\cos (\chi(t)) \omega_{\gamma}(t) & 0 & 0 \\
0 & 0 & 0 & 1 & 0 & 0 & 0 & 0 \\
0 & \omega_{\chi}(t) & 0 & 0 & 0 & -\sin (\chi(t)) \omega_{\gamma}(t) & 0 & 0 \\
0 & 0 & 0 & 0 & 0 & 1 & 0 & 0 \\
0 & 0 & 0 & \frac{\omega_{\gamma}(t)}{\sin (\chi(t))} & 0 & 0 & 0 & 0 \\
0 & 0 & 0 & 0 & 0 & 0 & 0 & 0 \\
0 & 0 & 0 & 0 & 0 & 0 & 0 & 0
\end{array}\right]}
\end{gathered}
$$


O processo de medição adotado tem uma natureza de tempo discreto, sendo aqui representado por $T_{k}$. A discretização da equação vetorial do processo (18), com período de amostragem $T_{k}$ foi feita utilizando a expansão em séries de Taylor em segunda ordem, sendo dado por (25):

$$
\hat{X}_{k+1 \mid k}=X_{k}+T_{k} \cdot \dot{X}_{k}+\frac{T_{k}^{2}}{2} \cdot \ddot{X}_{k}
$$

Na Equação (25) qual $X_{k}$ é o vetor de estados apresentado na Equação (19), $\dot{X}_{k}$ é a equação de (18) e $A_{k}$ é a matriz de transição de estados, dada por (23) ou (24), na forma discreta, tendo como base o instante de tempo $k$. O intervalo de tempo entre medições sucessivas, originárias de um mesmo objeto, é definido como $T_{k}=t_{k+1}-t_{k}$. O "^" representa valor estimado, dessa forma $\hat{X}_{k+1 \mid k}$ é o valor de $X$ estimado no instante de tempo $(k+1)$ com base no valor do instante anterior $k$. Assim, (18) na forma discreta passa a ser escrita como 26. $\ddot{X}_{k}$ na equação 27) representa a derivada da equação 26 em relação a $k$, para $\mathrm{U}$ constante.

$$
\begin{gathered}
\dot{X}_{k}=A \cdot X_{k}+U \\
\ddot{X}_{k}=A \cdot \dot{X}_{k}
\end{gathered}
$$

Substituindo as equações (26) e 27) em (25) obtemos a equação 28):

$$
\hat{X}_{k+1 \mid k}=\left(I+T_{k} \cdot A+\frac{T_{k}^{2}}{2} \cdot A^{2}\right) \cdot X_{k}+\left(I \cdot T_{k}+\frac{T_{k}^{2}}{2} \cdot A\right) \cdot U=F_{k} \cdot X_{k}+W_{k}
$$

Na Equação (28), I é a matriz identidade, e pode-se observar que na equação (28) temos os valores de $F_{k}$ e $W_{k}$ para os modelos CV e CT. Assim, para o modelo CV, substituindo estes $A_{k}$ de (23) e $U_{k}$ de (21) em (28), passamos a ter a equação de processo (29):

$$
\hat{X}_{k+1 \mid k}=\left[\begin{array}{cccccc}
1 & T_{k} & 0 & 0 & 0 & 0 \\
0 & 1 & 0 & 0 & 0 & 0 \\
0 & 0 & 1 & T_{k} & 0 & 0 \\
0 & 0 & 0 & 1 & 0 & 0 \\
0 & 0 & 0 & 0 & 1 & T_{k} \\
0 & 0 & 0 & 0 & 0 & 1
\end{array}\right] \cdot \hat{X}_{k \mid k}+\left[\begin{array}{ccc}
\frac{T_{k}^{2}}{2} & 0 & 0 \\
T_{k} & 0 & 0 \\
0 & \frac{T_{k}^{2}}{2} & 0 \\
0 & T_{k} & 0 \\
0 & 0 & \frac{T_{k}^{2}}{2} \\
0 & 0 & T_{k}
\end{array}\right] \cdot\left[\begin{array}{c}
w_{x} \\
w_{y} \\
w_{h}
\end{array}\right]
$$

Para o modelo CT, substituindo as equações (22) e (24) na Equação (28), é obtida a equação vetorial de processo para o modelo CT. Todavia, devido a componente $F_{k} \cdot X_{k}$ da equação (28) ser uma função de transição de estados não linear (que pode ser representada por $f\left(k, X_{k}\right)$ ), utilizamos o processo de linearização descrito em [5] para tratar o problema da não linearidade. Dessa forma, na função $f\left(k, X_{k}\right)$ foi aplicado o Jacobiano, sendo obtida a matriz $F_{l k}$ linearizada. Assim, a equação de processo linearizada para o modelo $\mathrm{CT}\left(\hat{X}_{k+1 \mid k}\right)$ é representada por 30 , onde alguns de seus componentes seguem destacados devido limitação de espaço: $a_{16}=b_{23}=-\cos \left(\chi_{k}\right) \omega_{\gamma k} \frac{T_{k}^{2}}{2}$; $a_{24}=-\omega_{\chi k} T_{k}-\cos \left(\chi_{k}\right) \frac{\omega_{\gamma}^{2} k}{\sin \left(\chi_{k}\right)} \frac{T_{k}^{2}}{2} ; a_{26}=-\cos \left(\chi_{k}\right) \omega_{\gamma k} T_{k}+\sin \left(\chi_{k}\right) \omega_{\chi k} \omega_{\gamma k} \frac{T_{k}^{2}}{2} ; a_{36}=b_{43}=$ $-\sin \left(\chi_{k}\right) \omega_{\gamma k} \frac{T_{k}^{2}}{2} ; a_{44}=1-\left(\omega_{\chi}^{2} k+\omega_{\gamma}^{2} k\right) \frac{T_{k}^{2}}{2} ; a_{46}=-\sin \left(\chi_{k}\right) \omega_{\gamma k} T_{k}-\cos \left(\chi_{k}\right) \omega_{\chi k} \omega_{\gamma k} \frac{T_{k}^{2}}{2} ; \mathrm{e}$ $a_{66}=1-\omega_{\gamma}^{2} k \frac{T_{k}^{2}}{2}$. 


$$
\begin{aligned}
& \hat{X}_{k+1 \mid k}=\left[\begin{array}{cccccccc}
1 & T_{k} & 0 & -\omega_{\chi k} \frac{T_{k}^{2}}{2} & 0 & a_{16} & 0 & 0 \\
0 & 1-\omega_{\chi}^{2} k \frac{T_{k}^{2}}{2} & 0 & a_{24} & 0 & a_{26} & 0 & 0 \\
0 & \omega_{\chi k} \frac{T_{k}^{2}}{2} & 1 & T_{k} & 0 & a_{36} & 0 & 0 \\
0 & \omega_{\chi k} T_{k} & 0 & a_{44} & 0 & a_{46} & 0 & 0 \\
0 & 0 & 0 & \frac{\omega_{\gamma k}}{\sin \left(\chi_{k}\right)} \frac{T_{k}^{2}}{2} & 1 & T_{k} & 0 & 0 \\
0 & \frac{\omega_{\gamma k} \omega_{\chi k}}{\sin \left(\chi_{k}\right)} \frac{T_{k}^{2}}{2} & 0 & \frac{\omega_{\gamma k}}{\sin \left(\chi_{k}\right)} T_{k} & 0 & a_{66} & 0 & 0 \\
0 & 0 & 0 & 0 & 0 & 0 & 1 & 0 \\
0 & 0 & 0 & 0 & 0 & 0 & 0 & 1
\end{array}\right] \cdot \hat{X}_{k \mid k} \\
& +\left[\begin{array}{ccccc}
\frac{T_{k}^{2}}{2} & 0 & 0 & 0 & 0 \\
T_{k} & -\omega_{\chi k} \cdot \frac{T_{k}^{2}}{2} & b_{23} & 0 & 0 \\
0 & \frac{T_{k}^{2}}{2} & 0 & 0 & 0 \\
\omega_{\chi k} \cdot \frac{T_{k}^{2}}{2} & T_{k} & b_{43} & 0 & 0 \\
0 & 0 & \frac{T_{k}^{2}}{2} & 0 & 0 \\
0 & \frac{\omega_{\gamma k}}{\sin \left(\chi_{k}\right)} \cdot \frac{T_{k}^{2}}{2} & T_{k} & 0 & 0 \\
0 & 0 & 0 & T_{k} & 0 \\
0 & 0 & 0 & 0 & T_{k}
\end{array}\right] \cdot\left[\begin{array}{c}
w_{x} \\
w_{y} \\
w_{h} \\
w_{\chi} \\
w_{\gamma}
\end{array}\right]
\end{aligned}
$$

Segundo [11, podemos assumir os ruídos $w_{x}, w_{y}, w_{h} w_{\chi}$ e $w_{\gamma}$ como independentes com média zero e variâncias $\sigma_{x}^{2}, \sigma_{y}^{2}, \sigma_{h}^{2}, \sigma_{\omega_{\chi}}^{2}$ e $\sigma_{\omega_{\gamma}}^{2}$ respectivamente. Para matriz de covariância dos ruídos associados ao estado $\left(Q_{k}\right)$, assumiremos $\sigma_{x}^{2}=\sigma_{y}^{2}=\sigma_{h}^{2}=\sigma_{q}^{2} \cdot Q_{k}$ é obtido fazendo: $Q_{k}=E\left[W_{k} \cdot W_{k}^{T}\right]$.

\subsection{Definição da Equação de Medida}

O sistema radar fornece o vetor de medidas $\mathbf{z}_{k}$ em coordenadas polares. Para relacionar este vetor com o vetor de estados do alvo $\mathbf{x}_{k}$ (definido em coordenadas cartesianas), houve a necessidade de aplicar uma transformação não linear, de modo que $\mathbf{z}_{k}$ passasse a ser escrito como $\mathbf{z}_{k}=\left[\begin{array}{llllllll}\mathbf{x}_{k}^{\mathbf{z}} & \mathbf{v}_{x k}^{\mathbf{z}} & \mathbf{y}_{k}^{\mathbf{z}} & \mathbf{v}_{y k}^{\mathbf{z}} & \mathbf{h}_{k}^{\mathbf{z}} & \mathbf{v}_{h k}^{\mathbf{z}} & \omega_{\chi k}^{\mathbf{z}} & \omega_{\gamma k}^{\mathbf{z}}\end{array}\right]^{T}$, passando a medida a ter coordenadas cartesianas antes da entrada no filtro, e em seguida, devido à natureza não linear da transformação, houve a necessidade de utilização das equações do Filtro de Kalman Estendido, para tratar a não linearidade.

Por meio de uma função de transformação trigonométrica, as medidas de distância $\left(\mathbf{r}_{k}\right)$, ângulo de azimute $\left(\theta_{k}\right)$ e ângulo de elevação $\left(\phi_{k}\right)$, obtidas em um sistema de coordenadas polares, são calculados os componentes de posição em coordenadas cartesianas $(x, y$ e $h$ ) como apresentado em (31):

$$
\mathbf{x}_{k}^{\mathbf{z}}=\mathbf{r}_{k} \cdot \cos \left(\theta_{k}\right) \cdot \cos \left(\phi_{k}\right), \quad \mathbf{y}_{k}^{\mathbf{z}}=\mathbf{r}_{k} \cdot \sin \left(\theta_{k}\right) \cdot \cos \left(\phi_{k}\right) ; \quad \text { e } \quad \mathbf{h}_{k}^{\mathbf{z}}=\mathbf{r}_{k} \cdot \sin \left(\phi_{k}\right)
$$

Os dados obtidos de medições dos radares nunca são exatos, pois poderão conter erros inerentes que, em geral, não são previsíveis. Dessa forma, não é razoável exigir que uma função de aproximação coincida exatamente com os dados. Segundo [12 tal função introduziria oscilações que não estavam presentes originalmente. Surge então a necessidade 
de se determinar uma função que se adapte a um dado conjunto de pontos de uma série de medições inexatas. De acordo com [12], o Método dos Mínimos Quadrados (MMQ), é uma maneira de determinar uma função polinomial de aproximação. Este método consiste em encontrar a função que melhor se ajusta ao conjunto de pontos dados. O MMQ minimiza o erro resultante do ajustamento, através da soma dos quadrados das diferenças entre os valores tabelados e os valores obtidos pela aproximação. Como dados de entrada para o MMQ, foram utilizados os dados de medida de posição atual $\left(\mathbf{z}_{k}\right)$, e das duas posições anteriores $\left(\mathbf{z}_{k-1}\right.$ e $\left.\mathbf{z}_{k-2}\right)$ com o instante de tempo $\left(T_{k}\right)$ que estas medidas ocorrem.

Assim, temos o problema de aproximar cada um dos três conjuntos de pares ordenados $\left(M_{k}, T_{k}\right)$, onde $M_{k}=x_{k}, y_{k}$ e $z_{k} ; T_{k}$ é o momento de obtenção de cada amostra e $k=0,1$ e 2, por um polinômio $\left(P_{M}\right)$ do segundo grau descrito por (32):

$$
P_{M}(T)=\sum_{k=0}^{2} a_{k M} T^{k}, \quad \text { onde } M=x, y \text { e } z .
$$

Deve-se então encontrar as constantes $a_{0 M}, a_{1 M}$ e $a_{2 M}$ que minimizam a soma dos quadrados dos desvios $(E)$ dado por $(33)$ :

$$
\begin{aligned}
E= & \sum_{k=0}^{2}\left(M_{k}-P\left(T_{k}\right)\right)^{2} \\
= & \sum_{k=0}^{2}\left(M_{k}\right)^{2}-2 \sum_{k=0}^{2} P\left(T_{k}\right) \cdot M_{k}+ \\
& \sum_{k=0}^{2}\left(P\left(T_{k}\right)\right)^{2} \\
= & \sum_{k=0}^{2}\left(M_{k}\right)^{2}-2 \sum_{k=0}^{2}\left(\sum_{j=0}^{2} a_{j} T_{k}^{j}\right) \cdot M_{k}+ \\
& \sum_{k=0}^{2}\left(\sum_{j=0}^{2} a_{j} T_{k}^{j}\right)^{2} \\
= & \sum_{k=0}^{2}\left(M_{k}\right)^{2}-2 \sum_{j=0}^{2} a_{j} \cdot\left(\sum_{k=0}^{2} M_{k} T_{k}^{j}\right)+ \\
& \sum_{j=0}^{2} \sum_{n=0}^{2} a_{j} a_{n}\left(\sum_{k=0}^{2} T_{k}^{j+n}\right)
\end{aligned}
$$

Para que $E$ tenha um mínimo é necessário que atenda a (34) e (35):

$$
\begin{gathered}
\frac{\partial E}{\partial a_{j}}=0, \quad j=0,1 \text { e } 2 \\
\frac{\partial E}{\partial a_{j}}=-2 \sum_{k=0}^{2} M_{k} T_{k}^{j}+2 \sum_{n=0}^{2} a_{n} \sum_{k=0}^{2} T_{k}^{j+n}
\end{gathered}
$$

Consolidando (34) e (35), tem-se um sistema de $n+1$ (neste caso 3) incógnitas 
$a_{j}$ e $n+1$ equações (neste caso 3), chamadas equações normais, dado por (36):

$$
\sum_{n=0}^{2} a_{n} \sum_{k=0}^{2} T_{k}^{j+n}=\sum_{k=0}^{2} M_{k} T_{k}^{j}, \quad j=0,1 \text { e } 2
$$

Estas últimas equações podem ser escritas como um sistema (37):

$$
\left\{\begin{aligned}
\text { Para } j=0: & a_{0 M} \sum_{k=0}^{2} T_{k}^{0}+a_{1 M} \sum_{k=0}^{2} T_{k}^{1}+ \\
& a_{2 M} \sum_{k=0}^{2} T_{k}^{2}=\sum_{k=0}^{2} M_{k} T_{k}^{0} \\
\text { Para } j=1: & a_{0 M} \sum_{k=0}^{2} T_{k}^{1}+a_{1 M} \sum_{k=0}^{2} T_{k}^{2}+ \\
& a_{2 M} \sum_{k=0}^{2} T_{k}^{3}=\sum_{k=0}^{2} M_{k} T_{k}^{1} \\
\text { Para } j=2: & a_{0 M} \sum_{k=0}^{2} T_{k}^{2}+a_{1 M} \sum_{k=0}^{2} T_{k}^{3}+ \\
& a_{2 M} \sum_{k=0}^{2} T_{k}^{4}=\sum_{k=0}^{2} M_{k} T_{k}^{2}
\end{aligned}\right.
$$

Resolvendo o sistema descrito em (37), para os três valores de $M$, onde: $M$ e $M_{k}=x_{k}, y_{k}$ e $z_{k}$; e $k=0,1$ e 2 , encontramos os valores para as constantes $a_{0 M}, a_{1 M}$ e $a_{2 M}$ para as três coordenadas $(x, y, z)$. Assim, substituindo na equação (32) obtemos os três polinômios $\left(P_{M}\right)$ que descrevem de forma aproximada a posição do objeto em coordenadas cartesianas, em função do tempo. De posse desses polinômios aplicamos a derivada em função do tempo (38) para obtermos o polinômio que descreve as velocidades em coordenadas cartesianas:

$$
v_{M}=\frac{d\left(P_{M}(T)\right)}{d T}, \quad \text { onde } M=x, y \text { e } z
$$

Assim, obtemos o valor das velocidades em $x, y$ e $z$ para o vetor de medidas $\mathbf{z}_{k}$, substituindo o valor de $T$, da medida atual, nos polinômios de (38).

Os ângulos de azimute $(\chi)$ e elevação $(\gamma)$, foram obtidos com a projeção dos vetores velocidades. A obtenção desses ângulos permitiu deduzir a velocidade angular $\omega_{\chi k}^{\mathbf{z}}$ a partir da diferença entre o ângulo de azimute $(k)$ e $(k-1)$, divididos pelo tempo. Foi aplicado o mesmo procedimento para obtenção de $\omega_{\gamma k}^{\mathbf{z}}$, a partir de $\gamma$. Seguindo o mesmo princípio, as acelerações angulares que foram utilizadas na matriz $H_{k+1}$, foram obtidas a partir da diferença entre velocidade angular atual e a anterior, divididas pelo tempo entre as mesmas.

A transformação não linear do vetor de medidas utilizada é dada por $\mathbf{r}_{k}, \theta_{k}$ e $\phi_{k}$, definidos em $(39)$ :

$$
\begin{gathered}
\mathbf{r}_{k}=\sqrt{\mathbf{x}_{k}^{2}+\mathbf{y}_{k}^{2}+\mathbf{h}_{k}^{2}}, \quad \theta_{k}=\arctan \left(\frac{\mathbf{x}_{k}}{\mathbf{y}_{k}}\right) \quad \mathrm{e} \\
\phi_{k}=\arctan \left(\frac{\mathbf{h}_{k}}{\sqrt{\mathbf{x}_{k}^{2}+\mathbf{y}_{k}^{2}}}\right)
\end{gathered}
$$

Assim, a função vetorial não linear que relaciona as variáveis de estado às medidas, 
denotada por $f h\left(k+1, \mathbf{x}_{k+1}\right)$, é dada por 40):

$$
f h\left(k+1, \mathbf{x}_{k+1}\right)=\left[\begin{array}{c}
f h 1=\sqrt{\mathbf{x}_{k+1}^{2}+\mathbf{y}_{k+1}^{2}+\mathbf{h}_{k+1}^{2}} \\
f h 2=\arctan \left(\frac{\mathbf{x}_{k+1}}{\mathbf{y}_{k+1}}\right) \\
f h 3=\arctan \left(\frac{\mathbf{h}_{k+1}}{\sqrt{\mathbf{x}_{k+1}^{2}+\mathbf{y}_{k+1}^{2}}}\right) \\
f h 4=\omega_{\chi k}^{\mathbf{z}} \\
f h 5=\omega_{\gamma k}^{\mathbf{z}}
\end{array}\right]
$$

A matriz da covariância dos ruídos associados à medida $\left(R_{k+1}\right)$ adotada é dada por 41):

$$
R_{k+1}=\left[\begin{array}{ccccc}
\sigma_{\mathbf{r}} & 0 & 0 & 0 & 0 \\
0 & \sigma_{\theta} & 0 & 0 & 0 \\
0 & 0 & \sigma_{\phi} & 0 & 0 \\
0 & 0 & 0 & \sigma_{\dot{\omega}_{\chi}} & 0 \\
0 & 0 & 0 & 0 & \sigma_{\dot{\omega}_{\gamma}}
\end{array}\right]
$$

A linearização da função $f h\left(k+1, \hat{\mathbf{x}}_{k+1 \mid k}\right)$, foi através do cálculo da matriz jacobiana da mesma. A matriz resultante obtida foi denotada por $H_{k+1}$, sendo definida por (42):

$$
H_{k+1}=\left[\begin{array}{ccccc}
\frac{\mathbf{x}}{\sqrt{\mathbf{x}^{2}+\mathbf{y}^{2}+\mathbf{h}^{2}}} & \frac{\mathbf{y}}{\mathbf{x}^{2}+\mathbf{y}^{2}+\mathbf{h}^{2}} & \frac{-\mathbf{x h}}{\sqrt{\mathbf{x}^{2}+\mathbf{y}^{2}}\left(\mathbf{x}^{2}+\mathbf{y}^{2}+\mathbf{h}^{2}\right)} & 0 & 0 \\
0 & 0 & 0 & 0 & 0 \\
\frac{\mathbf{y}}{\sqrt{\mathbf{x}^{2}+\mathbf{y}^{2}+\mathbf{h}^{2}}} & \frac{-\mathbf{x}}{\mathbf{x}^{2}+\mathbf{y}^{2}+\mathbf{h}^{2}} & \frac{-\mathbf{y h}}{\sqrt{\mathbf{x}^{2}+\mathbf{y}^{2}}\left(\mathbf{x}^{2}+\mathbf{y}^{2}+\mathbf{h}^{2}\right)} & 0 & 0 \\
0 & 0 & 0 & 0 & 0 \\
\frac{\mathbf{x}}{\sqrt{\mathbf{x}^{2}+\mathbf{y}^{2}+\mathbf{h}^{2}}} & 0 & \frac{\sqrt{\mathbf{x}^{2}+\mathbf{y}^{2}}}{\mathbf{x}^{2}+\mathbf{y}^{2}+\mathbf{h}^{2}} & 0 & 0 \\
0 & 0 & 0 & 0 & 0 \\
0 & 0 & 0 & \dot{\omega}_{\chi} & 0 \\
0 & 0 & 0 & 0 & \dot{\omega}_{\gamma}
\end{array}\right]^{T}
$$

Para inicialização dos modelos, foram consideradas as duas primeiras amostras, conforme apresentado em [5]. Um método de inicializar a matriz de covariância é defini-la como $P_{0 \mid 0}=\alpha^{2} \cdot Q_{k}$, sendo o valor típico de $\alpha=10$. $Q_{k}$ representa a covariância dos erros associados ao processo.

O ruído de processo foi adotado como sendo branco na aceleração para os modelos utilizados. O desvio padrão do ruído de processo $\left(\sigma_{q}\right)$ para o modelo CV foi definido como em [5], $\sigma_{q}=\alpha \cdot a_{\max }$, onde $0<\alpha \leq 1$ e $a_{\max }$ é a aceleração máxima. Foram definidos empiricamente $\alpha=0.7$ e $a_{\max }=5 \mathrm{~m} / \mathrm{s}^{2}$, com isso, $\sigma_{q}=3,5 \mathrm{~m} / \mathrm{s}^{2}$. No modelo CT o nível de ruído é maior que no modelo $\mathrm{CV}$, pois a incerteza é maior no momento das manobras. Foram definidos empiricamente $\alpha=0.8, a_{\max }=125 \mathrm{~m} / \mathrm{s}^{2}$, o desvio padrão do ruído do processo $\left(\sigma_{q}=100 \mathrm{~m} / \mathrm{s}^{2}\right)$, os ruídos nas taxas de giro como $\sigma_{\omega_{\chi}}=\sigma_{\omega_{\gamma}}=0.044 \mathrm{rad} / \mathrm{s}^{2}$, os ruídos das taxas de aceleração angular $\left(\sigma_{\dot{\omega}_{\chi}}=\sigma_{\dot{\omega}_{\gamma}}=5 \mathrm{rad} / \mathrm{s}^{2}\right)$ para o CT. Os ruídos de medida dependem diretamente das características do sensor modelado. Nas trajetórias em que estes ruídos foram considerados, utilizou-se um desvio padrão na distância de 
$\sigma_{\mathbf{r}}=75 \mathrm{~m}$, no ângulo de azimute de $\sigma_{\theta}=0.0175^{\circ}$ e no ângulo de elevação de $\sigma_{\phi}=0.0175^{\circ}$, como em [8].

Para a definição dos elementos da matriz de transição (П) do algoritmo IMM, foi adotado o intervalo de tempo para obtenção de cada amostra de 2 segundos, o tempo estimado para a permanência de deslocamento de forma linear de 200 segundos (CV) e o tempo estimado para uma trajetória não linear de 20 segundos (CT), assim П é dado por 43):

$$
\Pi=\left[\begin{array}{cc}
0.99 & 0.01 \\
0.1 & 0.9
\end{array}\right]
$$

\section{TESTES E RESULTADOS}

Para avaliar o modelo, foi realizado um comparativo com alguns resultados apresentados em [8] e [13]. Estes foram escolhidos para comparação com os resultados deste trabalho, por apresentarem modelos tridimensionais com uma técnica inovadora para cálculo da velocidade angular e com testes descritos em um nível de detalhamento suficiente para permitir sua reprodução e comparação. As trajetórias comparadas com [8] são tipo ziguezague, formadas por sete trechos, como na Figura 2 (a).

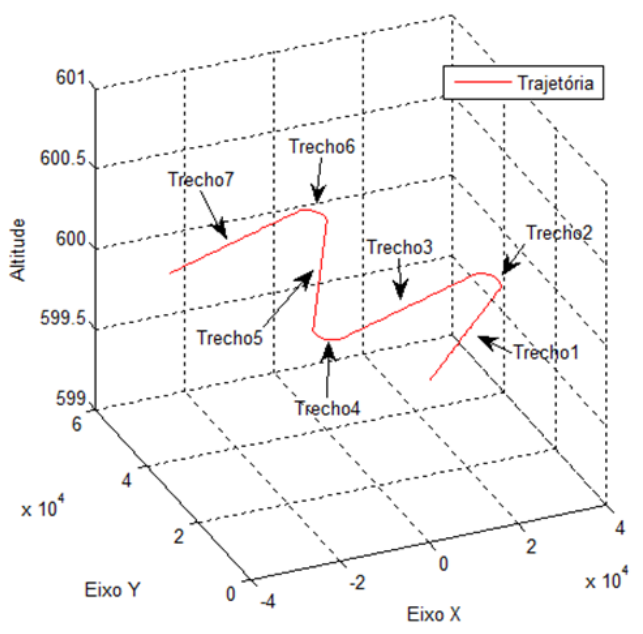

(a)

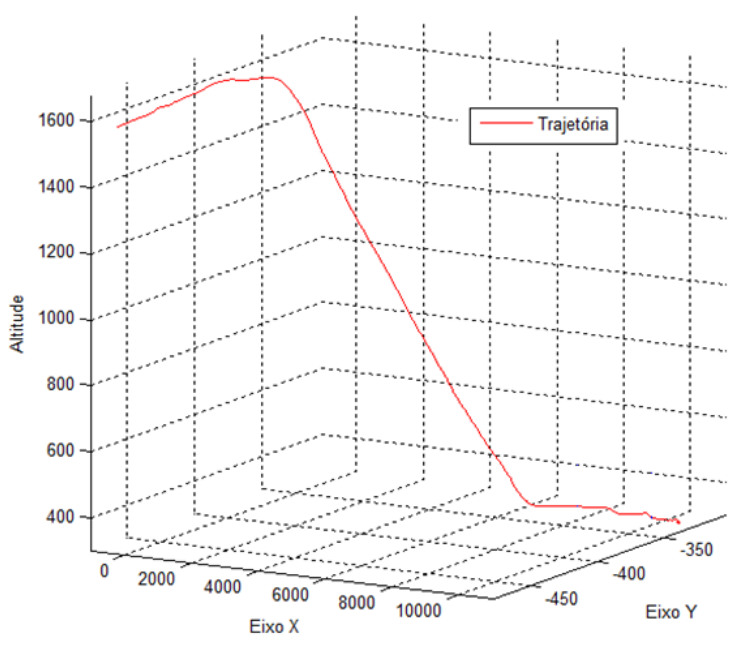

(b)

Figura 2: (a)Trajetória Manobra Zigue-zague. (b)Trajetória real com alteração de altitude.

O critério utilizado para avaliar este trabalho foi o RMS (erro médio quadrático) obtido pela comparação entre a trajetória prevista pelo modelo em cada instante com a posição real futura do objeto, para os diferentes deslocamentos realizados com evolução no tempo para o vetor de posições, sendo dado por (44):

$$
\operatorname{RMS}_{k}^{i}=\sqrt{\frac{1}{k}\left(r e s_{k}^{i}\right)^{T} r e s_{k}^{i}}, \quad k=1,2, \ldots, n ; \quad i=x, y \text { ou } h
$$

Onde $k$ é a ordem de contagem das amostras, $i$ são os eixos cartesianos, $r e s_{k}^{i} \in \mathbf{R}$ são os vetores resíduos calculados pelas diferenças entre a posição da amostra e a estimativa 
de posição calculada pelos modelos, e $n$ é o número de dados da trajetória ou do trecho da trajetória. A partir dos $n$ valores obtidos de $\mathrm{RMS}_{k}^{i}$, calculou-se o RMS médio para cada componente de posição, dado por (45):

$$
\overline{\operatorname{RMS}}_{i}=\frac{\sum_{k=1}^{n} \operatorname{RMS}_{k}^{i}}{n}, \quad k=1,2, \ldots, n ; \quad i=x, y \text { ou } h
$$

Os critérios adotados por [8], também aqui utilizados, foram a média dos $\overline{\mathrm{RMS}}_{i}$ 45), e a norma de $\overline{\mathrm{RMS}}$ 46):

$$
\|\overline{\mathrm{RMS}}\|_{x y z}=\sqrt{\overline{\mathrm{RMS}}_{x}^{2}+\overline{\mathrm{RMS}}_{y}^{2}+\overline{\mathrm{RMS}}_{z}^{2}}
$$

Para que fosse possível efetuar a comparação entre os trabalhos, adotou-se os mesmos parâmetros de ajustes citados em [8]. Sendo assim, $T=4 s, \sigma_{r}{ }^{2}=75^{2}, \sigma_{\chi}{ }^{2}=$ $0,0175^{2}$ e $\sigma_{\gamma}^{2}=0,0175^{2}$, em que $T$ é o período de amostragem (intervalo de tempo entre as amostras), $\sigma_{r}{ }^{2}$ é a variância do alcance radar, $\sigma_{\chi}{ }^{2}$ é a variância do ângulo de azimute e $\sigma_{\gamma}^{2}$ é a variância do ângulo de elevação. Assim como em [8], considerouse a estimativa inicial de estado sendo dada por $\hat{\mathbf{x}}_{0 \mid 0}=\left[\begin{array}{l}-30000 \mathrm{~m} \\ 196,16 \mathrm{~m} / \mathrm{s}-\end{array}\right.$ $30000 \mathrm{~m} \quad 154,98 \mathrm{~m} / \mathrm{s} \quad 600 \quad 0 \quad 0 \quad 0]^{T}$.

Uma das trajetórias utilizadas para comparação com [8] foi a de um plano com inclinação vertical de $30^{\circ}$ em relação ao plano horizontal $x y$.

Comparando (a) e (b) da Figura 3, o modelo deste trabalho obteve um desempenho superior ao de [8], tanto para cada um dos sete trechos apresentados quanto para a trajetória como um todo.

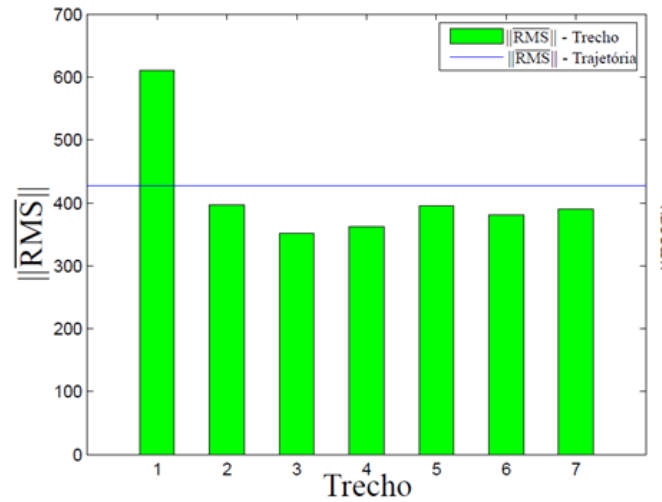

(a)

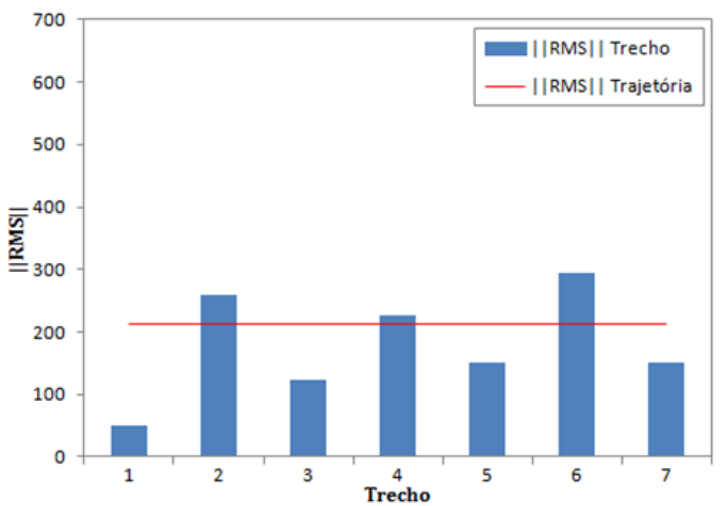

(b)

Figura 3: Gráfico de $\|\overline{\mathrm{RMS}}\|$. Inclinado $30^{\circ}$. (a)Fonte: [8. (b)modelos deste trabalho.

A outra forma utilizada para avaliar o a efetividade do modelo descrito neste trabalho foi a comparação com dados reais. Foram realizadas simulações para comparação entre o modelo apresentado, utilizando as hipóteses adotadas para os ruídos descritas anteriormente, com sistema real utilizado em Navios da Marinha do Brasil (MB). Os dados reais da trajetória das aeronaves utilizados nos testes foram obtidos dos testes da Avaliação Operacional (AO) de um navio da MB. Os testes da AO se destinavam a verificar o resultado de solução de tiro para o disparo de um projetil de canhão de 4,5 
polegadas, ao acompanhar aeronaves. Utilizou-se como referência para origem o sistema cartesiano $(\mathrm{x}, \mathrm{y}, \mathrm{h})$. As trajetórias foram percorridas pelas aeronaves com uma velocidade em torno de $360 \mathrm{~km} / \mathrm{h}$. As duas primeiras trajetórias foram percorrendo um trajeto com movimento vertical ascendente (ilustrada na Figura 2 (b)), e a terceira percorrendo um trajeto com movimento de alteração de rumo horizontal. A primeira possuía posição inicial $(7647,43 ;-325,31 ; 315,83)$ e final $(-699,03 ;-462,62 ; 1571,95)$. A segunda possuía posição inicial $(-5466,93 ;-3010,67 ; 285,87)$ e final $(1797,83 ;-3026,43 ; 1552,44)$. A terceira possuía posição inicial $(6376,27 ;-191,34 ; 313,88)$ e final $(1118,47 ;-6900,68 ; 289,83)$. Para a primeira trajetória, a comparação entre resultados mostrou uma melhora de 88,995\% no desempenho do modelo proposto quando comparado aos dados obtidos na AO. Para a segunda mostrou uma melhoria de $87,838 \%$ e para a terceira uma melhoria de $72,307 \%$ ).

\section{CONCLUSÕES}

Para tratar a previsão de posição de objetos com mudança no seu perfil de deslocamento, foram escolhidos os modelos de Velocidade Constante e Giro Constante, que são baseados no sistema de coordenadas cartesianas. Com base nestes modelos bidimensionais, foram deduzidos, os modelos CV e CT tridimensionais, com a conversão do sistema de coordenadas das amostras obtidas de coordenadas esféricas para coordenadas cartesianas.

Analisando os resultados dos testes realizados com estes modelos, apresentados na Seção 4 é possível verificar que tanto o CV como o CT tridimensionais, foram capazes de estimar de forma satisfatória uma posição seguinte com base nas posições das amostras anteriores para todas as trajetórias testadas.

O modelo CT apresentado é o mais significativo, pois ele pode trabalhar em planos arbitrários do espaço, aumentando a possibilidade de conseguir manter uma previsão de posição dos objetos durante seu deslocamento de forma adequada. A trajetória descrita pelo objeto acompanhado pode estar contida em um plano paralelo ao formado pelos eixos $x$ e $y$, mas que é rotacionada no eixo $z$.

A forma de se obter as informações das velocidades escalares de cada eixo cartesiano, para fazerem parte do vetor de observações $\mathbf{z}_{k}$ foram descritas. As velocidades foram deduzidas a partir das informações da medida atual e duas medidas anteriores, com o auxílio do método dos Mínimos Quadrados Discretos. Os ângulos de azimute e elevação do alvo, em cada amostragem, foram obtidos a partir da projeção destes vetores velocidades. Assim, a velocidade angular $\omega_{\chi}$ pôde ser obtida a partir da diferença entre o ângulo de azimute do instante da amostra $k$ e da amostra anterior, divididos pelo tempo entre as amostras. Foi aplicado o mesmo procedimento para obtenção de $\omega_{\gamma}$, a partir do ângulo de elevação. No modelo de observações foram incluídas duas informações deduzidas, as velocidades angulares do ângulo de azimute e de elevação na matriz $H_{k+1}$ da equação de medição, que relaciona o estado à medida.

A abordagem proposta foi validada pelos resultados dos testes apresentados, os quais foram comparados com os resultados de outros trabalhos da literatura e também utilizando-se de dados reais obtidos da MB. Assim, a principal contribuição deste trabalho é o desenvolvimento de uma nova abordagem para cálculo do modelo $\mathrm{CT}$, através das coordenadas cartesianas de forma tridimensional.

Como trabalhos futuros é proposta a conversão dos modelos implementados neste trabalho em MATLAB, para uma linguagem de baixo nível (como C). Essa conversão é 
importante, pois para o uso dos modelos em sistemas especialistas, com a utilização de dados reais, é necessária para uma avaliação mais consistente do algorítimo, possibilitando realizar melhorias em sua implementação. Além disso, para melhorar a análise do problema, do modelo e seu desempenho em uma aplicação prática, é interessante tratar o problema do tempo de amostra variável, pois neste trabalho a amostragem foi realizada com tempo fixo. Maiores detalhes podem ser obtidos em [1].

\section{REFERÊNCIAS BIBLIOGRÁFICAS}

[1] JUNIOR, J. A. Acompanhamento de múltiplos alvos em trajetórias tridimensionais, 2016, xvii, 113p. Dissertação (Mestrado) - Programa de Pós-Graduação de Modelagem Computacional, Laboratório Nacional de Computação Científica, Petrópolis, 2016. 2. 15

[2] FRENCL, V. B.; VAL, J. B. do. Tracking with range rate measurements: Turn rate estimation and particle filtering. In: IEEE. Radar Conference (RADAR), 2012 IEEE. [S.1.], 2012. p. 0287-0292. 2

[3] YUAN, X.; LIAN, F.; HAN, C. Models and algorithms for tracking target with coordinated turn motion. Mathematical Problems in Engineering, Hindawi Publishing Corporation, v. 2014, 2014. 2

[4] FRENCL, V. B.; VAL, J. B. do. A trajectory simulator for individual movements using probabilistic distribution profiles. In: IEEE. Radar Conference (RadarConf), 2016 IEEE. [S.1.], 2016. p. 1-6. 2

[5] BAR-SHALOM, Y.; LI, X. R.; KIRUBARAJAN, T. Estimation with applications to tracking and navigation: theory algorithms and software. [S.1.]: John Wiley \& Sons, 2001. 2, 3, 7, 11

[6] KORBICZ, J. et al. Fault diagnosis. Models, artificial intelligence, applications. [S.l.]: Springer Berlin/Heidelberg, 2004. 2, 5

[7] BAR-SHALOM, Y. et al. Mathematics in science and engineering. [S.l.]: Academic press, 1988. 3

[8] FRENCL, V. B. Técnicas de filtragem utilizando processos com saltos markovianos aplicados ao rastreamento de alvos móveis. Dissertação (Mestrado) - Universidade Estadual de Campinas (UNICAMP). Faculdade de Engenharia Elétrica e de Computação, 2010. 3, 12, 13

[9] AGUIRRE, L. Introdução à Identificação de Sistemas - Técnicas Lineares e NãoLineares Aplicadas a Sistemas Reais. [S.l.]: Editora UFMG, 2007. ISBN 9788570415844. 3

[10] WELCH, G.; BISHOP, G. Course 8-an introduction to the kalman filter. SIGGRAPH 2001 Courses, 2001. 3

[11] KANG, E. Radar System Analysis, Design, and Simulation. [S.l.]: Artech House, 2008. (The Artech House radar library). ISBN 9781596933477. 8

[12] BURDEN, R. L.; FAIRES, J. D.; TASKS, A. Análise numérica. [S.1.]: Cengage Learning, 2008. 8, 9 
[13] FRENCL, V. B.; VAL, J. B. R. do. Rastreamento utilizando filtro de partículas com perturbação gaussiana na estimativa e medidas de velocidade radial. 2012. 12 